\title{
542 Characteristic Variations of Chip Morphology and Cutting Forces in Face Milling with Flat-Faced and Grooved Tool Inserts
}

\author{
Ning Fang \\ Department of Mechanical and Aerospace Engineering \\ Utah State University, Logan, Utah 84322-4130, U. S. A., nfang@mae.usu.edu \\ Web: http://www. mae.usu.edu/faculty/nfang
}

\begin{abstract}
The modeling of face milling operations has been traditionally focused on using flat-faced tools with an assumption of a perfectly straight chip formation. However, a curled chip formation is a common phenomenon in practical milling operations, and it has a profound effect on machining performances. A new analytical model that takes into account the chip curling effect in milling operations is presented in this paper. It is shown that chip morphology and machining parameters, such as the chip up-curl radius, the chip thickness, and the toolchip contact length, simultaneously change with varying undeformed chip thickness during each tooth cycle. The model is validated through the milling tests. A good agreement between theory and experiments has been reached. A comparison of the average resultant force is made among three types of commercially available tool inserts to demonstrate the effect of tool insert chip-groove design on milling performances.
\end{abstract}

Key Words: Face Milling, Slip-Line Model, Chip Morphology, Cutting Forces

\section{INTRODUCTION}

Face milling is among the most effective methods of manufacturing high accuracy products with relatively low cost and high productivity. The modeling of face milling operations has been an important research topic highly motivated by industrial demands, e.g., the monitoring, planning, and optimization of milling operations. Various methods such as the mechanistic approach [1-2], Oxley's machining theory [3], the stability analysis [4-5], and the finite element analysis [6] have been widely employed. Milling chips are often assumed perfectly straight to simplify theoretical models.

However, a curled chip formation is a common phenomenon in practical milling. It is necessary to take into account the chip curling effect. The reason can be explained as follows: (1) Theoretically, a good understanding of chip curl helps understand the mechanism of chip formation. A large amount of study $[7,8]$ has demonstrated that a curled chip formation has a significant effect on machining performances, e.g., the cutting forces, cutting temperatures, tool wear, and tool life. (2) Practically, it helps optimize the tool geometry to achieve better chip control in milling. Milling has been traditionally conducted using flat-faced tools. Grooved tools have been predominately employed in continuous machining like turning. Not a long time ago, it was realized that grooved tools also provide favorable conditions for reduced cutting forces and tool wear and improved machining accuracy in intermittent machining such as milling [9]. At present, grooved tools for milling applications are commercially available. Compared to the rapid development of milling practices, the theoretical study of milling with grooved tools has largely lagged behind.
A new analytical model for face milling operations is presented in this paper based on a recently developed universal slip-line model [10] involving a curled chip formation. The mathematical formulation of the new model is illustrated first. Then, characteristic variations of chip morphology and machining parameters in face milling operations are analyzed. The new model is validated through the milling tests. A good agreement between theory and experiments is reached. Finally, a comparison of the average resultant force is made among three types of commercially available tool inserts to demonstrate the effect of chip-groove design on milling performances.

\section{A NEW ANALYTICAL MODEL}

\subsection{Face milling operations}

Figure 1 shows the face milling operation investigated in this paper. A face milling cutter with a diameter of $D$ is used to cut a work material. The machine tool spindle rotational speed is $n$. The milling cutter entry and exit angle are denoted by $\alpha_{01}$ and $\alpha_{02}$, respectively. The feed rate per tooth is $f_{z}$, and the axial depth of cut is $a_{p}$. The variable $\theta_{t}$ determines the position of the milling cutter at time $t$ during each tooth cycle. Let $t=0$ when $\theta_{t}=\alpha_{01}$.

Three cutting force components, $F_{x}, F_{y}$, and $F_{z}$, are directly measured by a dynamometer. Through a simple mathematical transformation, these force components can be translated into the tangential force $F_{T}$, the radial force $F_{R}$, and the axial force $F_{A}$.

\subsection{Instantaneous chip side-flow angle $\eta_{s}(t)$}

One of the most prominent characteristics of milling operations is the undeformed chip thickness 

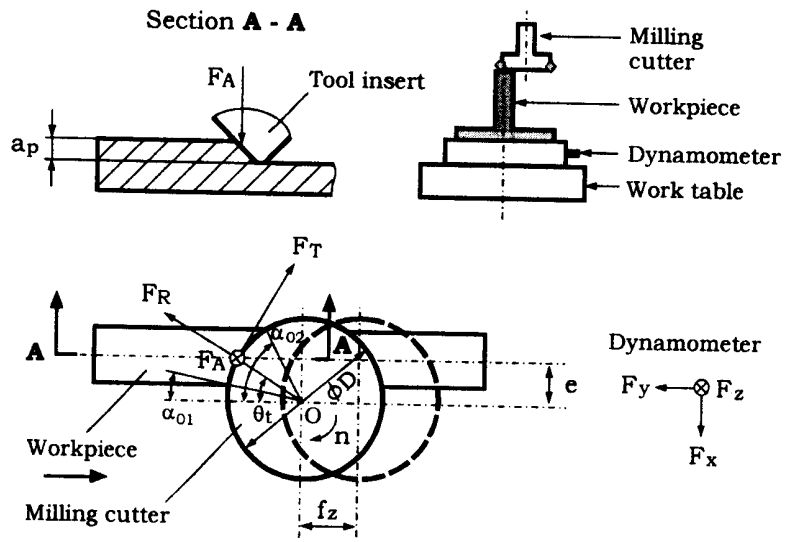

Fig. 1 Schematic diagram of face milling

$h_{c}$ keeps changing during each tooth cycle, or $h_{c}$ is a function of time $t$, denoted by $h_{c}(t)$. In this paper, a variable $X$ is denoted by $X(t)$ if it is time dependent.

The instantaneous feed rate $f(t)$ for each tooth is determined by

$$
f(t)=f_{z} \cdot \cos \left(\alpha_{O 1}+2 \pi n \cdot t\right)
$$

Thus, the undeformed chip thickness $h_{c}(t)$ can be calculated as

$$
h_{c}(t)=f(t) \cdot \sin \kappa_{r}
$$

where $\kappa_{r}$ is the tool cutting edge angle.

Figure 2 shows the chip side-flow on the tool rake face, where $\lambda_{s}$ is the tool inclination angle, $\gamma_{r}$ is the tool radial rake angle, and $V_{c h}(t)$ is the instantaneous chip flow speed.
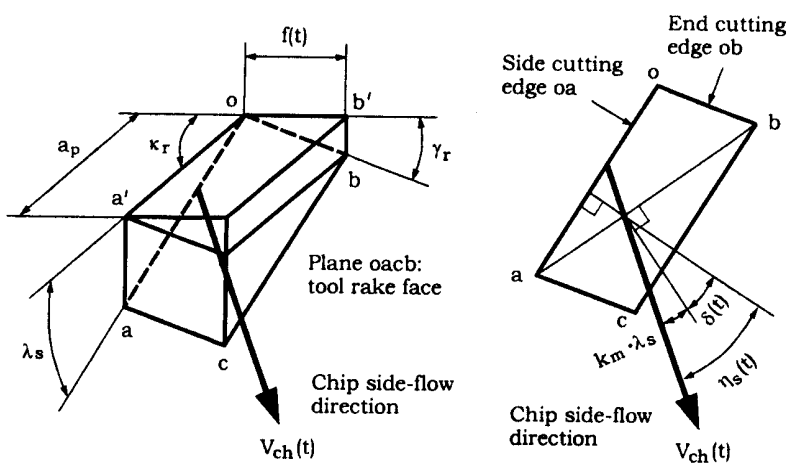

Fig. 2 Chip side-flow on the tool rake face

From geometric relationships shown in Fig. 2, the following equations hold:

$$
\begin{aligned}
& o a=\frac{a_{p}}{\sin \kappa_{r} \cdot \cos \lambda_{s}} \\
& o b(t)=f(t) / \cos \gamma_{r}
\end{aligned}
$$

The instantaneous chip side-flow angle $\eta_{s}(t)$ is calculated as

$$
\eta_{s}(t)=k_{m} \cdot \lambda_{\mathrm{s}}+\delta(t)
$$

where $k_{m}$ is a material constant, $\delta(t)$ is expressed as

$$
\delta(t)=\tan ^{-1} \frac{o b(t) \cdot \sin \kappa_{r}}{o a+o b(t) \cdot \cos \kappa_{r}}
$$

The first term $k_{m} \cdot \lambda_{s}$ on the right-hand side of Eq. (5) is based on Stabler's law [11] and indicates the effects of work material and tool inclination angle on the chip side-flow. The second term $\delta(t)$ indicates the effects of cutting conditions (the feed rate and the depth of cut) and tool geometry (the tool cutting edge angle and the tool radial rake angle).

\subsection{Variation of the tool geometry}

The tool axial rake angle $\gamma_{a}$ is given by [12]

$$
\gamma_{a}=\tan ^{-1} \frac{\tan \lambda_{s}+\tan \gamma_{r} \cdot \cos \kappa_{r}}{\sin \kappa_{r}}
$$

The tool normal rake angle $\gamma_{n}$ is expressed as

$$
\begin{array}{r}
\gamma_{n}=\tan ^{-1}\left[\operatorname { c o s } \lambda _ { s } \cdot \left(\tan \gamma_{r} \cdot \sin \kappa_{r}\right.\right. \\
\left.\left.+\tan \gamma_{a} \cdot \cos \kappa_{r}\right)\right]
\end{array}
$$

Measured in a plane $P_{e}$ parallel to the chip sideflow direction and perpendicular to the tool rake face, the effective tool rake angle $\gamma_{e}(t)$ is given by

$$
\begin{aligned}
\gamma_{e}(t)=\sin ^{-1}\left[\sin \lambda_{s} \cdot \sin \eta_{s}(t)\right. \\
\left.+\cos \lambda_{s} \cdot \sin \gamma_{n} \cdot \cos \eta_{s}(t)\right]
\end{aligned}
$$

For flat-faced tools with a land (or chamfer) length of $h$, the effective land length $h_{e}(t)$ measured in the said plane $P_{e}$ is calculated as

$$
h_{e}(t)=h / \cos \eta_{s}(t)
$$

\subsection{Instantaneous chip up-curl radius $R_{u}(t)$, chip} thickness $h_{c h}(t)$, and tool-chip contact length $\boldsymbol{l}_{\boldsymbol{n}}(t)$

Based on the rigid-plastic and plane-strain assumptions, a universal slip-line model [10] was recently developed for machining with restricted contact tools as shown in Fig. 3 , where $A C$ is a convex upward shear plane; $\theta, \psi, \eta_{1}$, and $\eta_{2}$ are four slip-line angles; $V_{c}$ is the cutting speed; and $\gamma_{1}$ is the tool side rake angle. The model takes into account both the chip up-curling and chip back-flow effects and incorporates six slip-line models previously developed for machining during the last six decades, i.e., Dewhurst's model [13] (as shown in Fig. 4), Shi and Ramalingam's model, Kudo's model, Johnson's and Usui and Hoshi's models, Lee and Shaffer's model, and Merchant's model, as special cases [10]. The universal slip-line model has been validated through extensive cutting tests covering a wide range of cutting conditions $[14,15]$.

The universal slip-line model [10] is extended to investigate face milling in the present study. Chip deformation keeps changing at each time instant $t$ during each tooth cycle in milling. It is assumed that for each chip deformation at each time instant $t$, the universal slip-line model is applicable in the said plane $P_{e}$ as long as the axial depth of cut $a_{p}$ is at least 10 times larger than the undeformed chip thickness $h_{c}(t)$, i.e., as long as the plane-strain conditions of chip deformation is satisfied.

Dewhurst and Collins' [16] matrix technique for numerically solving slip-line problems has been employed in the mathematical formulation of the 

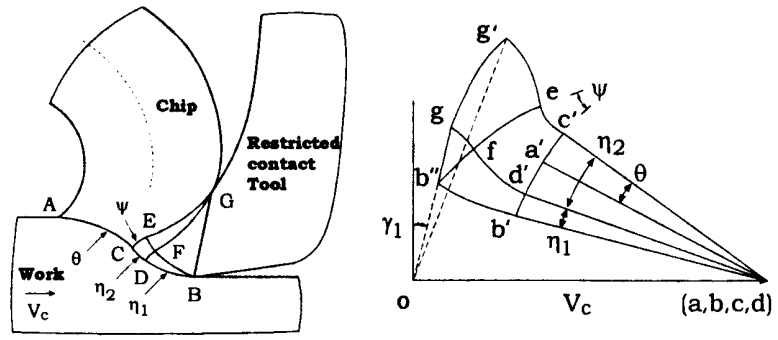

Fig. 3 Universal slip-line model (left) and its hodograph (right) [10]
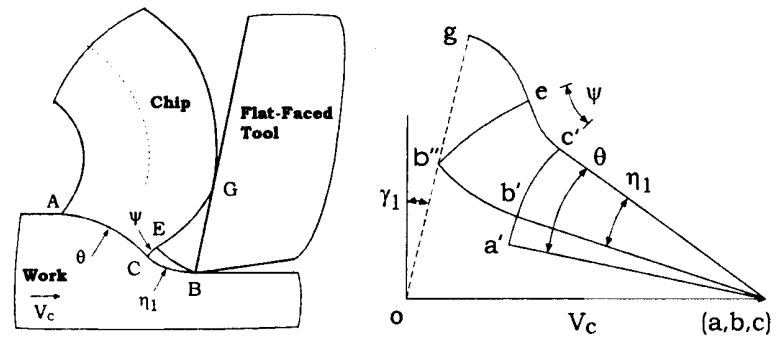

Fig. 4 A special case: Dewhurst's model (left) and its hodograph (right) [13]

slip-line model. The slip-line $G E$ is taken as the base slip-line, and its radius of curvature denoted by a column vector $\vec{\sigma}$ is given by

$$
\vec{A} \cdot \vec{\sigma}=\vec{B} \cdot[\rho(t) / \omega(t)] \cdot \vec{c}
$$

where $\rho(t)$ is the magnitude of velocity jump across slip-line $A C D B, \omega(t)$ is the angular velocity of curled chip rotation, $\vec{C}$ is a column vector, and $\vec{A}$ and $\vec{B}$ are expressed as [10]

$$
\begin{gathered}
\vec{A}=\vec{I}-\left(\vec{P}_{\eta_{1} \eta_{2}} \vec{R}_{\eta_{1}} \vec{G}_{\zeta} \vec{Q}_{\psi \eta_{1}} \vec{P}_{\eta_{2}}^{*}+\vec{Q}_{\eta_{2} \eta_{1}} \vec{Q}_{\psi \eta_{2}}\right) \cdot \\
\left(\vec{P}_{\psi \eta_{2}} \vec{Q}_{\eta_{1}}^{*} \vec{G}_{\zeta} \vec{P}_{\eta_{2}}^{*}+\vec{Q}_{\eta_{2} \psi} \vec{Q}_{\eta_{1} \eta_{2}}\right) \\
\vec{B}=\vec{P}_{\eta_{1} \eta_{2}} \vec{R}_{\eta_{1}} \vec{G}_{\zeta}\left(\vec{P}_{\eta_{1} \psi}+\vec{Q}_{\psi \eta_{1}} \vec{Q}_{\eta_{2}}^{*}\right) \\
+\vec{Q}_{\eta_{2} \eta_{1}} \vec{P}_{\eta_{2} \psi}
\end{gathered}
$$

where $\vec{P}, \vec{P}^{*}, \vec{Q}, \vec{Q}^{*}, \vec{R}$, and $\vec{G}_{\zeta}$ are members of a set of basic matrix operators defined by Dewhurst and Collins [16], and $I$ is a unit vector.

For machining with flat-faced tools, inputs of the slip-line model include the tool side rake angle $\gamma_{1}$, the hydrostatic pressure ratio $P_{A} / k_{A B}$, and the frictional stress ratio $\tau / k_{\text {int }}$, where $P_{A}$ is the hydrostatic pressure at point $A$ (see Fig. 3 or 4 ), $k_{A B}$ is the material shear flow stress along the shear plane $A B, \tau$ is the frictional shear stress at the toolchip interface, and $k_{\text {int }}$ is the material shear flow stress at the tool-chip interface $[10,13]$.

Figure 5 shows the effective tool and chip geometry measured along the chip side-flow direction in the said plane $P_{e}$, where $R_{u}(t), h_{c h}(t)$, and $l_{n}(t)$ are instantaneous chip up-curl radius, chip thickness, and tool-chip contact length, respectively; and $\phi_{e}(t)$ is the equivalent shear-plane angle [13].

The instantaneous chip up-curl radius $R_{u}(t)$ is

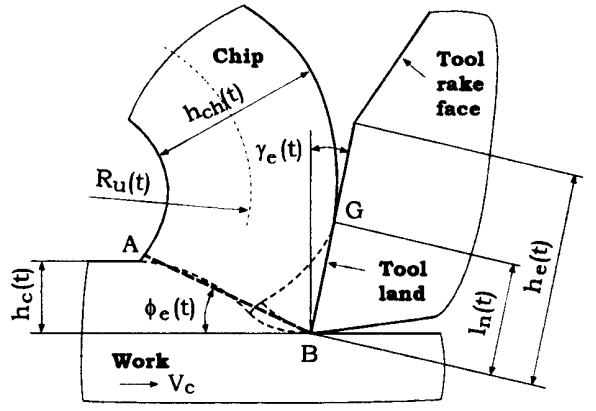

Fig. 5 Effective tool and chip geometry measured along the chip side-flow direction in the plane $P_{e}$

determined by

$$
\begin{aligned}
R_{u}(t)= & \frac{1}{2} \sqrt{\left(\frac{V_{c}}{\omega(t)}\right)^{2}+\left(\frac{\rho(t)}{\omega(t)}\right)^{2}+2 \cdot \frac{V_{c}}{\omega(t)} \cdot \frac{\rho(t)}{\omega(t)} \cdot \cos \alpha_{1}(t)} \\
& +\frac{V_{g^{\prime}}(t)}{2 \omega(t)}
\end{aligned}
$$

where $\alpha_{l}(t)$ is the angle of the slip-line $A C$ with the work material free surface, and $V_{g}(t)$ is the chip velocity at the moment of departing from the tool rake face.

The instantaneous chip thickness $h_{c h}(t)$ is expressed as

$$
h_{c h}(t)=2\left[\frac{V_{g^{\prime}}(t)}{\omega(t)}-R_{u}(t)\right]
$$

For milling with flat-faced tools, the instantaneous tool-chip contact length $\ln (t)$, i.e., the length $B G$ in Fig. 4, is calculated as

$$
l_{n}(t)=\frac{\sqrt{\left[X_{e-g e}(t)-Y_{e-b e}(t)\right]^{2}-}}{\left[X_{e-b e}(t)+Y_{e-g e}(t)\right]^{2}}
$$

where $\left[X_{e-g e}(t), Y_{e-g e}(t)\right]$ are Cartesian coordinates of Point $E$ on the slip-line $G E$ with the base point at $G$, and $\left[X_{e-b e}(t), Y_{e-b e}(t)\right]$ are Cartesian coordinates of Point $E$ on the slip-line $B E$ with the base point at $B$.

\subsection{Instantaneous chip width $b_{c h}(t)$ and chip length $l_{\text {ch }}(t)$}

The instantaneous chip width $b_{c h}(t)$ is determined by

$$
b_{c h}(t)=\frac{a_{p} \cdot \cos \eta_{s}(t)}{\sin \kappa_{r} \cdot \cos \lambda_{s}}
$$

The equivalent shear-plane angle $\phi_{e}(t)$ is calculated as

$$
\phi_{\mathrm{e}}(t)=\tan ^{-1} \frac{\cos \gamma_{e}(t)}{h_{c h}(t) / h_{c}(t)-\cos \gamma_{e}(t)}
$$

The instantaneous chip flow speed $V_{c h}(t)$ is determined by

$$
V_{c h}(t)=V_{c} \cdot \frac{\sin \phi_{\mathrm{e}}(t)}{\cos \left[\gamma_{e}(t)-\phi_{\mathrm{e}}(t)\right]}
$$

The instantaneous chip length $l_{c h}(t)$ is given by

$$
l_{c h}(t)=\int_{O}^{t} V_{c h}(t) \cdot \mathrm{d} t
$$




\section{THE EXPERIENTAL SET-UP}

\subsection{Face milling tests}

A face milling cutter with indexable tool inserts was used to cut AISI 4140 low alloy steel, as shown in Fig. 1. The cutting speed $\left(V_{c}\right)$ kept constant at $160 \mathrm{~m} / \mathrm{min}$. The feed rate per tooth $\left(f_{z}\right)$ varied between 0.025 and $0.153 \mathrm{~mm} / \mathrm{rev}$. The axial depth of cut $\left(a_{p}\right)$ varied between 0.4 and $2.0 \mathrm{~mm}$.

\subsection{Tool inserts employed}

Three types of commercially available tool inserts were investigated. One type (AZ) has a flat rake face. The other two types (WL and WM) have a built-in complex chip-groove on the tool rake face. Figure 6 shows their appearance and a cross section perpendicular to the tool side cutting edge based on the tool-in-hand system. The actual (working) tool geometry changes in the tool-in-use system.
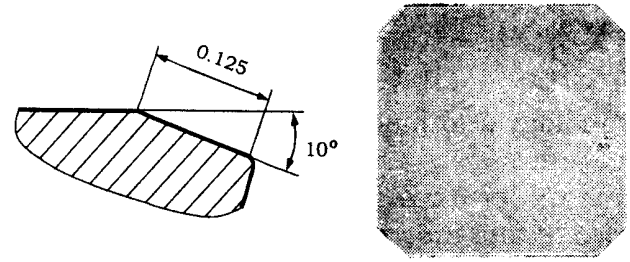

(1) AZ-type flat-faced insert
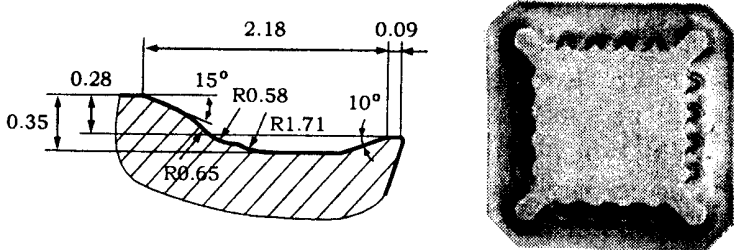

(2) WL-type grooved insert
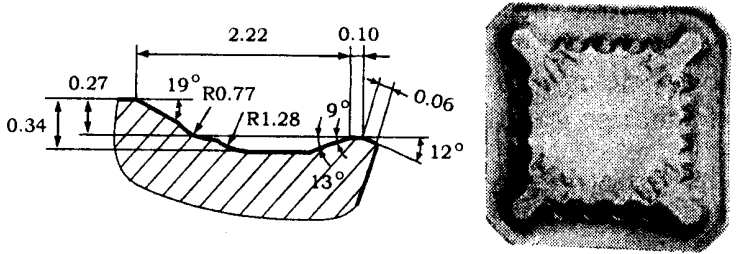

(3) WM-type grooved insert

Fig. 6 Tool inserts employed in the cutting tests

\subsection{Measurement of the cutting forces}

The cutting force measurement system consists of a Kistler type 9257B Quartz 3-component dynamometer, a Kistler type 5001B charge amplifier, and a computer data acquisition system.

\section{ANALYSIS AND EXPERIMENTAL VALIDATION OF THE ANALYTICAL MODEL}

\subsection{Inputs of the analytical model}

AZ-type flat-faced inserts with a curled chip formation are analyzed. In the tool-in-use system,
AZ-type inserts have a tool inclination angle $\lambda_{s}$ of $19^{\circ}$ and a tool radial rake angle of $1^{\circ}$ on its chamfer [see Fig. 6(1)]. If the feed rate per tooth $f_{z}$ is far less than the chamfer length $h(0.125 \mathrm{~mm})$, the tool-chip contact occurs only on the tool chamfer, which is the machining case shown in Fig. 5. Inputs of the analytical model described in Section 2 include:

(1) Tool geometry: tool inclination angle $\lambda_{s}=19^{\circ}$ tool cutting edge angle $\kappa_{r}=45^{\circ}$ tool radial rake angle $\gamma_{r}=1^{\circ}$ tool land (chamfer) length $h=0.125 \mathrm{~mm}$

(2) Cutting conditions:

cutting speed $V_{c}=160 \mathrm{~m} / \mathrm{min}$ spindle rotational speed $n=800 \mathrm{rpm}$ feed rate per tooth $f_{z}=0.077 \mathrm{~mm} / \mathrm{rev}$. axial depth of cut $a_{p}=2 \mathrm{~mm}$ milling cutter entry angle $\alpha_{01}=8.21^{\circ}$ cutting time $t=0 \sim 0.017 \mathrm{~s}$

(3) Stabler's constant of chip flow $k_{m}=0.9$

(4) Stress boundary conditions of the slip-line field: hydrostatic pressure ratio $P_{A} / k_{A B}=0.9$ frictional stress ratio $\tau / k_{\text {int }}=0.98$

\subsection{Characteristic variations of chip morphology and machining parameters}

Figure 7 shows characteristic variations of chip morphology and machining parameters. It can be seen clearly from Fig. 7 that the undeformed chip thickness $h_{c}(t)$, the tool-chip contact length $l_{n}(t)$, the chip thickness $h_{c h}(t)$, and the chip up-curl radius $R_{u}(t)$ decrease with increasing time $t$ during each tooth cycle in face milling. However, the chip sideflow angle $\eta_{s}(t)$, the chip flow speed $V_{c h}(t)$, the effective tool rake angle $\gamma_{e}(t)$, the effective tool land length $h_{e}(t)$, and the chip width $b_{c h}(t)$ remain almost constant.

\subsection{Experimental validation of chip morphology}

Figure 8 shows the morphology of a chip generated during face milling with AZ-type tool inserts under the same cutting conditions given in Section 4.1. Figure 8 clearly shows the variation of chip up-curl radius, which is in agreement with the predicted results shown in Fig. 7(5).

The curled chip is spread out as flat as possible to carefully measure its thickness at a $2 \mathrm{~mm}$ interval along the chip length. The measurement is repeated three times for each chip, and the average chip thickness is taken as the experimental data. Figure 9 shows the comparison between the predicted and experimental chip thickness. As seen clearly, the predicted value agrees well with its experimental value. The predicted chip length is also very close to its measured value. The measurement also shows that the chip width keeps almost constant along the chip length, which agrees well with the predicted results shown in Fig. 7(6). 


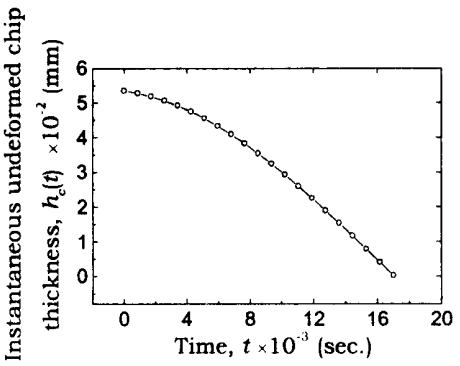

(1) $h_{c}(t)$ vs. $t$

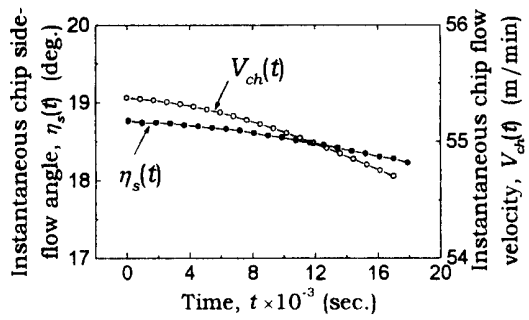

(2) $\eta_{s}(t)$ and $V_{c h}(t)$ vs. $t$

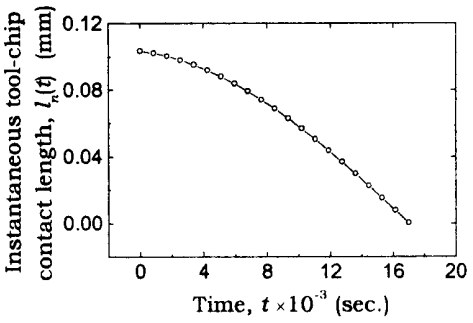

(3) $l_{n}(t)$ vs. $t$

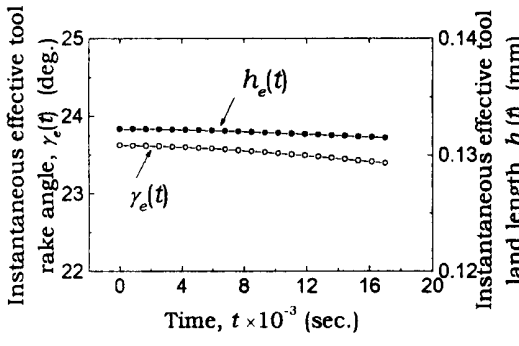

(4) $\gamma_{e}(t)$ and $h_{e}(t)$ vs. $t$

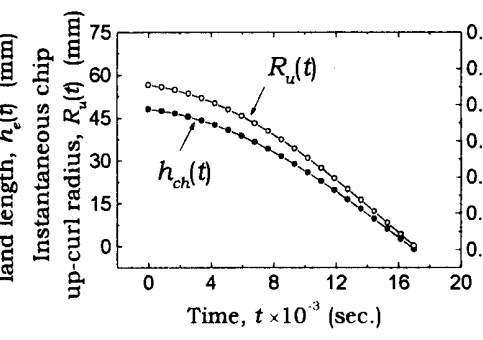

(5) $R_{u}(t)$ and $h_{c h}(t)$ vs. $t$

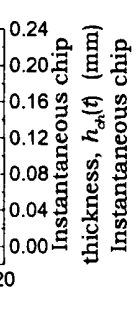

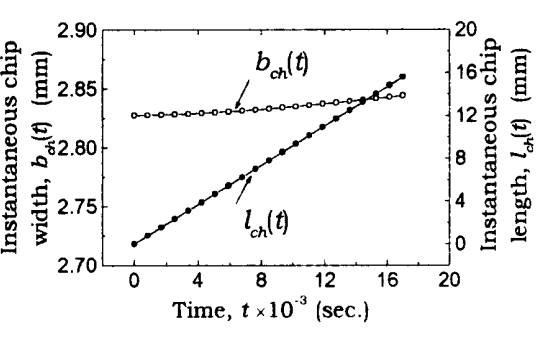

(6) $b_{\operatorname{ch}}(t)$ and $\operatorname{lch}(t)$ vs. $t$

Fig. 7 Characteristic variations of chip morphology and machining parameters

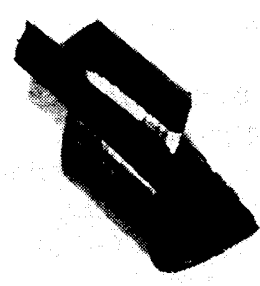

Fig. 8 A typical chip morphology in face milling

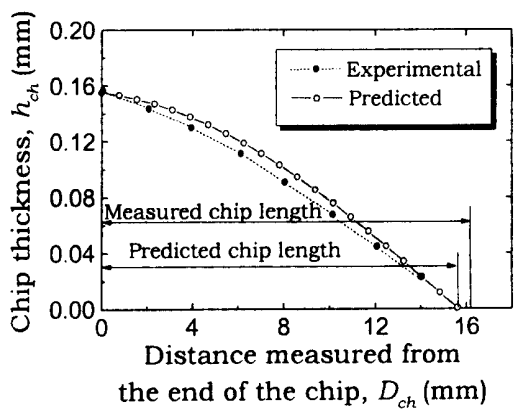

Fig. 9 Comparison of the predicted and experimental chip thickness

\section{COMPARISON OF THE FORCE BETWEEN FLAT-FACED AND GROOVED TOOL INSERTS}

The resultant force keeps changing at each time instant during each tooth cycle in milling. For comparison purposes, the average resultant force $F$ was employed in the present study. A total of 63 cutting tests have been conducted. Figures 10 and 11 show the comparison of the average resultant force under a range of cutting conditions with three types of tool inserts shown in Fig. 6.

As seen from Figs. 10 and 11, WL-type grooved inserts produce the lowest forces under almost all of the tested cutting conditions. Compared with AZtype flat-faced inserts, WL-type grooved inserts reduce the average resultant force by as much as $27 \%$ (!) in the best case. However, the performance of WM-type grooved inserts seems complex. Depending on specific cutting conditions, WM-type grooved inserts may generate higher or lower forces than AZ-type flat-faced inserts.

The observation made above well demonstrates that the tool insert chip-groove design significantly affects milling performances. An optimized chipgroove design with the effective chip control performance greatly benefits milling operations.

\section{CONCLUDING REMARKS}

The major contributions of this paper include:

(1) A new analytical model that takes into account the chip curling effect is presented for face milling. The model has been successfully employed to study characteristic variations of chip morphology and machining parameters. The new model is validated through the milling tests. A good agreement between theory and experiments has been reached.

(2) Through a large amount of experimental evidence, it is well demonstrated that milling performances can be greatly improved through the effective tool insert chip-groove design. The extension of the traditional chip control study from continuous machining (turning) to intermittent machining (milling) opens a new and promising research area. 


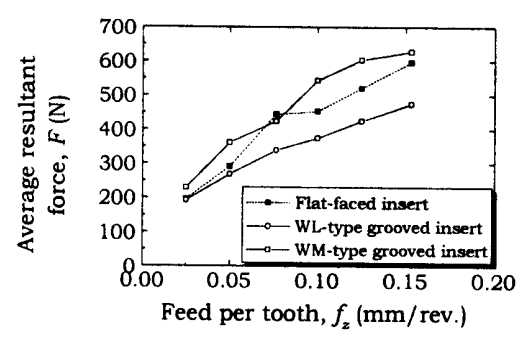

(1) $a_{p}=2.0 \mathrm{~mm}$

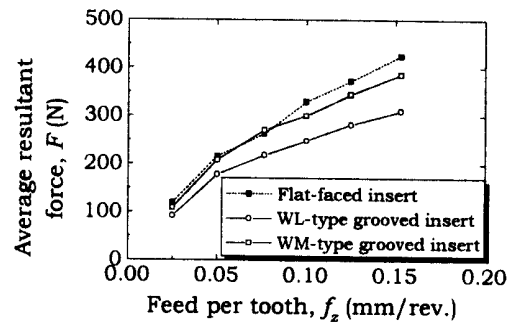

(2) $a_{p}=1.2 \mathrm{~mm}$

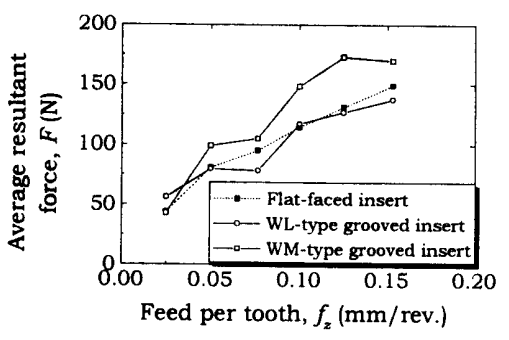

(3) $a_{p}=0.4 \mathrm{~mm}$

Fig. 10 Comparison of the average resultant force at different axial depths of cut $a_{p}$

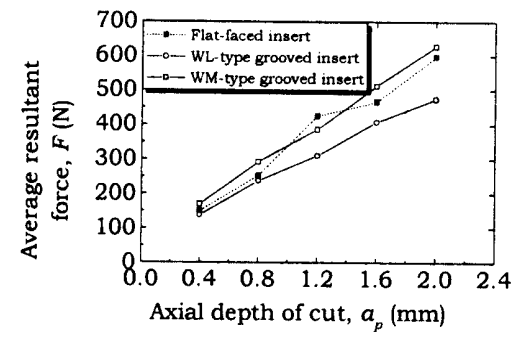

(1) $f_{z}=0.077 \mathrm{~mm} / \mathrm{rev}$.

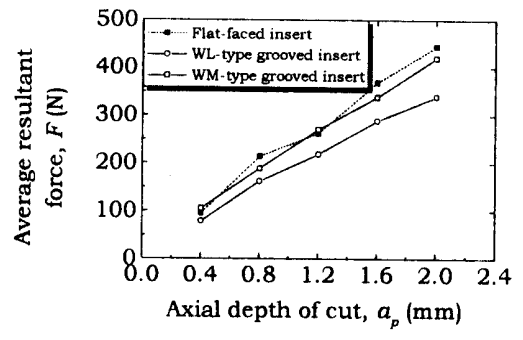

(2) $f_{z}=0.05 \mathrm{~mm} / \mathrm{rev}$.

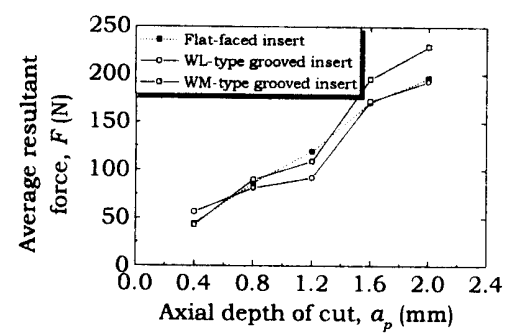

(3) $f_{z}=0.025 \mathrm{~mm} / \mathrm{rev}$.

Fig. 11 Comparison of the average resultant force at different feed rates $f_{z}$

\section{REFERENCES}

[1] Gu, F., Kapoor, S.G., DeVor, R.E. and Bandyopadhyay, P., An Enhanced Cutting Force Model for Face Milling with Variable Cutter Feed Motion and Complex Workpiece Geometry, J. Manufact. Sci. Engg., Vol. 119, (1997), p467

[2] Altintas, Y., Modeling Approaches and Software for Predicting the Performance of Milling Operations at MAL-UBC, Proc. of 3th CIRP Int. Workshop on Modelling of Machining Operations, Sydney, Australia, (2000), p60

[3] Zheng, H. Q., Li, X.P., Wong, Y.S. and Nee, A.Y.C., Theoretical Modeling and Simulation of Cutting Forces in Face Milling with Cutter Runout, Int. J. Machine Tools \& Manufact., Vol. 39, (1999), p2003

[4] Jensen, S.A. and Shin, Y.C., Stability Analysis in Face Milling Operations, Part 2: Experimental Validation and Influencing Factors, J. Manufact. Sci. Engg., Vol. 121, (1999), p606

[5] Smith, S. and Tlusty, J., Update on High-Speed Milling Dynamics, J Engg. For Industry, Vol. 112, (1990), p142

[6] Ueda, K., Manabe, K. and Nozaki, S., RigidPlastic FEM Analysis of Three-Dimensional Cutting Mechanism ( $2^{\text {nd }}$ Report)-Simulation of Plain Milling Process, J. Japan Soc. Prec. Engg,, (in Japanese), Vol. 62, (1996), p526

[7] Van Luttervelt, C.A., Childs, T.H.C., Jawahir, I.S., Klocke, F. and Venuvinod, P.K., Present Situation and Future Trends in Modeling of Machining Operations, Vol. 47, (1998), p587

[8] Obikawa, T., Shinozuka, J. and Shirakashi, T., Analytical Prediction of Cutting Performances of
Grooved Rake Face Tools, J. Japan Soc. Prec. Engg., (in Japanese), Vol. 61, (1995), P1295

[9] Wertheim, R. and Satran, A., The Development of New Insert Geometries and Chip Formers for Milling Applications, Canadian Machinery \& Metalworking, Vol. 90, (1995), p21

[10] Fang, N., Jawahir, I.S. and Oxley, P.L.B., A Universal Slip-Line Model with Non-Unique Solutions for Machining with Curled Chip Formation and a Restricted Contact Tool, Int. J. Mech. Sci., Vol. 43, (2001), p557

[11] Stabler, G.V., The Fundamental Geometry of Cutting Tools, Proc. Inst. Mech. Engrs., Vol. 165, (1951), p14

[12] Fang, N., An Improved Model for Oblique Cutting and Its Application to Chip Control Research, J. Mater. Proc. Techn., Vol. 79, (1998), p79

[13] Dewhurst, P., On the Non-Uniqueness of the Machining Process, Proceedings of Royal Society of London, Series A, Vol. 360, (1978), p587

[14] Fang, N. and Jawahir, I.S., An Analytical Predictive Model and Experimental Validation for Machining with Grooved Tools Incorporating the Effects of Strains, Strain-Rates, and Temperatures, Annals of CIRP, to appear in Vol. 51, (2002)

[15] Fang, N. and Jawahir, I.S., Analytical Predictions and Experimental Validation of Cutting Forces, Chip Thickness, and Chip Back-Flow Angle in Restricted Contact Machining Using the Universal Slip-Line Model, Int. J. Machine Tools \& Manufact. Vol. 42, (2002), p681

[16] Dewhurst, P. and Collins, I.F., A Matrix Technique Constructing Slip-Line Field Solutions to a Class of Plane Strain Plasticity Problems, Int. J. for Num. Methods in Engg., Vol. 7, (1973), p357 